The objection to the ase of these derivatives of the suprarenal gland is that their effect lasts only for a few minutes, three or four; this is, however, readily overcome by continnous injection, which may be made to last some hours,

\section{FrG. 8.}
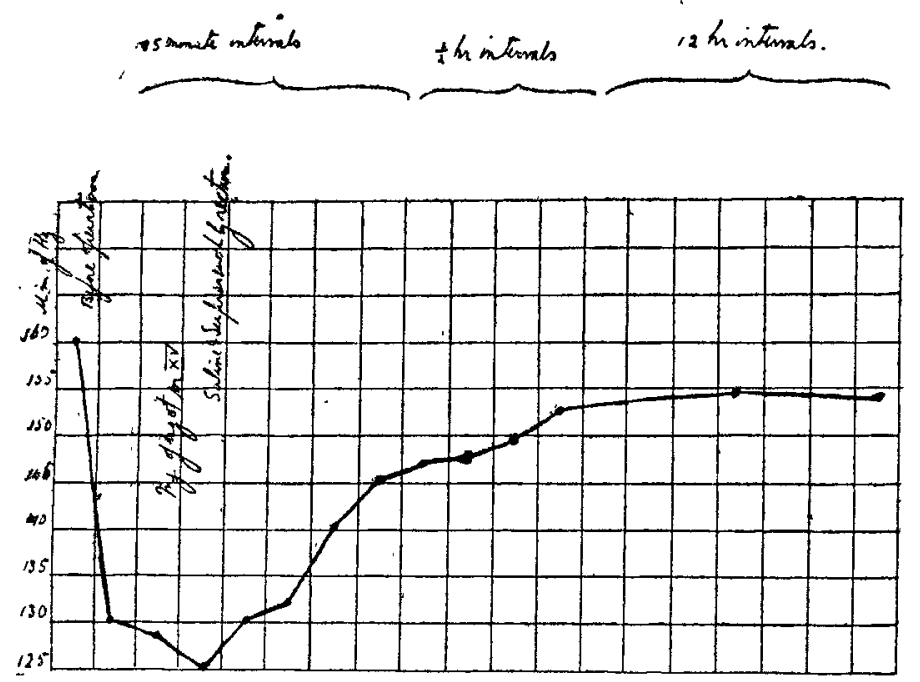

Patient aged 25 years. Strangulation of gut by diverticulum. Severe shock. Patient was given ergot hypodermically and continuous rectal infusions of saline solution and suprarenal extract 1-160000th Oi. per hour, also injections of morphine 1 grain. The rise in pressure after the suprarenal is well marked.

FIG. 9.

$$
\text { is num mationils }
$$

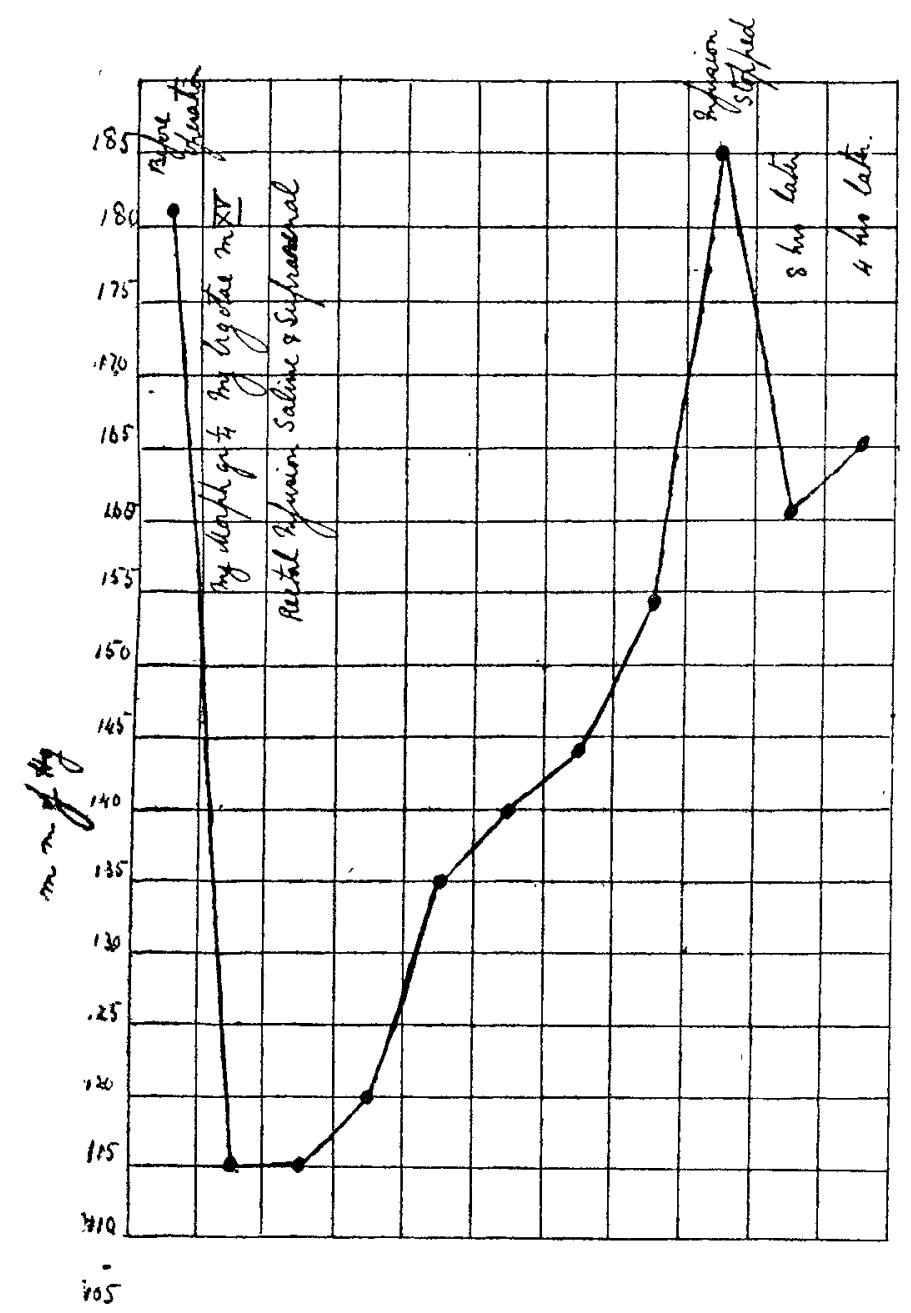

Patient aged 52 years. Amputation of breast, removal of axillary glands. Severe shock. Patient was given ergot hypodermically, and continuous rectal infusion of saline solution and suprarenal extract 1-160000th Oi. per hour, also injections of morphine $\frac{1}{4}$ grain. The marked fall in pressure from the operation, and the steady rise, even to above normal, are well shown.

and thus the effects of the drug maintained until the condition of shock has passed off, and as it produces its effects even when given in small doses, sufficient is not administered even by these continuous methods to give rise to any toxic symptoms. Orile advises that it should be given in a $1-50000$ or 1-100000 solution. The strength $\mathrm{I}$ prefer is $1-160000$, that is one drachm to the pint of the solution sold. This is a dilution easy to remember and to make up, and appears to be sufficiently strong for its purpose. The dilution is made with normal saline solution.

As regards the method of administration, intravenously is perhaps the best physiologically, but it necessitates the formation of a new wound with after-pain to the patient, and it is more difficult to manage. When the fluid is given for some hours it must be left in charge of a nurse, to whom the methods of dealing with blockage of the tubes, leakage, \&c., often present considerable difficulties. Subcutaneous injection has the same objections, with the addition that the insertion of a large quantity of fluid here, even if given slowly, may cause considerable pain, and, if the solution be too strong, may even cause sloughing of the overlying skin. In one case where the solution was given 1-20000, an area about six inches by four inches sloughed out of the inner side of each thigh, and necessitated subsequent skin grafting. Continuous rectal injection presents none of these difficulties. A rubber tube is arranged as a syphon from the bowl of fluid, which is raised about one foot above the level of the patient. At the other end of the tube is connected a glass vaginal nozzle, which is passed three or four inches up the rectum, and the fluid, at a temperature of from $108^{\circ}$ to $112^{\circ} \mathrm{F}$., is allowed to run in at the rate of one pint an hour. The only objection that can be raised is that the absorption of the extract does not take place, but I have already shown that this objection does not seem to hold good.

'Ergot.-The effects of this drug are very similar to those of the suprarenal products, but they last considerably longer. Lockhart Mummery, ${ }^{1}$ experimenting with it upon a cat, showed that a steady rise was caused which was maintained for 20 minutes. The objections to it are that its preparations are uncertain; a useful and fairly reliable one is the B.P. hypodermic injections, as made at the dispensary of this hospital-15 minims will be found a useful dose.

Morphine.-As already mentioned, the anæsthetic, by decreasing the pain and the afferent impulses to the vaso-motor centres, decreases the amount of shock. As the effect of the anresthetic passes off and the pain of the wound becomes again appreciable it would be expected that the shock would be more marked, and such is often found to be the case; Figs. 5, 6, and 7 show a steady fall of pressure after the operation has ceased. By giving morphine this appreciation of pain is much decreased and the condition of shock is not maintained; morphine is also well known as a cardiac stimulant and is thus of use for this effect.

(To be concluded.)

\section{EXTIRPATION OF THE LACRYMAL SAC.}

\section{BY O. J. CURRIE, M.B. LOND. \& CAPE UNIV.,} M.R.C.S. ENG.,

SURGEON TO GREY'S HOSPITAL, PIETERMARITZBURG.

THE lacrymal sac is deeply situated at the lower and anterior part of the inner wall of the orbit, resting on the lacrymal groove. It forms the upper dilated end of the lacrymo-nasal duct, which drains the conjunctival sac and conducts superfluous tears into the nasal cavity.

Dacryocystitis, or inflammation of the lacrymal sac, may be acute or chronic. Acute cases should be treated with hot fomentations, pus should be evacuated if an abscess forms, and the sac should be syringed out through the incision or through the upper or lower canaliculus. Probing the nasal duct should not be resorted to until after syringing out the sac has been given a trial, and not in any case until the acute symptoms have subsided. Chronic dacryocystitis should be treated as follows. First, by syringing out the sac with antiseptic and astringent lotions, such as solutions. of boric acid, chinosol, or argyrol; secondly, if perseverance in the above treatment fails, by slitting the uppes or lower canaliculus and dilating the nasal duct with probes: and thirdly, in neglected or intractable cases which cannot be cured by the above means, destruction or extirpation of the lacrymal sac is necessary. 
Theobald mentions the following surgical procedures for the cure of dacryocystitis: (1) The restoration of the natural passages; (2) the formation of a new passage into the nose; (3) the obliteration of the natural passages, the lacrymal sac and duct; and (4) the removal of the lacrymal gland. Desmarres recommended the third of these proceedings only when the case was incurable by other means. Theobald agrees with this and recommends the use of largesized probes as a means of restoring the patency of the nasal duct.

H. Knapp gives the following indications for extirpations of the lacrymal sac, viz. :-In all cases where an important lacrymal disease can otherwise not so well or not at all be cured, as in the following conditions: (1) Large mucocele; (2) chronic lacrymal fistula; (3) fibrous or osseous occlusion of the nasal duct with lacrymation and discharge; (4) chronic stricture of the nasal duct with lacrymation and discharge ; (5) chronic dacryocystoblennorrhœea with degeneration of the walls of the sac; and (6) chronic catarrhal dacryocystitis with repeated attacks of phlegmonous inflammation.

Although destruction of the lacrymal sac by the actual cautery in cases of lacrymal fistula appears to have been performed in the days of Celsus it was not till 1724 that the more surgical procedure of extirpation by the knife was performed by Platner, and there still seems to be some divergence of opinion among surgeons as to the indications for the operation and also as to whether the knife or the cautery should be employed. I think it is now generally recognised that the operation might be more frequently performed than it is in cases of dacryocystitis, which, owing to neglect of the patient or perhaps unwise or too vigorous treatment in the early stages, have lapsed into a state of chronic suppuration. The old treatment by caustics or the cautery is now being rejected in favour of the cleaner and more surgical proceeding of extirpation.

The objections urged against the operation are its dangers and difficulties. The chief danger is orbital cellulitis and its complications, which can be avoided by careful asepsis and by drainage of the wound. The sac must be frequently syringed out with an antiseptic lotion for some days before the operation in order to diminish the risk of infection of the wound, and drainage by means of a few strands of catgut or a fine strip of gauze should certainly be employed in order to give exit to any blood or serum which might form a nidus for the growth of pathogenic germs in the depths of the wound.

The difficulties of the operation arise from the deep situation of the sac beneath the tendo oculi and from the field of view being obscured by hæmorrhage. The former can be readily overcome by making the incision sufficiently free and by the use of suitable retractors, such as Axenfeld's, and the latter is now avoided by sponging out the wound with adrenalin solution ( 1 in 1000).

It is a curious fact that complete removal of the lacrymal sac results in a cure of the epiphora. There are two theories to account for this apparent paradox. 1. Physiological sympathy induces the gland to cease secreting. 2. That under normal conditions the amount of tears secreted by the gland is only sufficient to keep the conjunctiva moist and that in health, unless under exceptional circumstances, such as emotion or irritation of the conjunctiva, there is no excess of tears and no overflow down the nasal duct. Probably both these hypotheses are correct, as it is stated that if a portion of the lacrymal sac is accidentally left behind it excites a reflex secretion of tears and keeps up watering of the eye. The following case, which has recently been under my care, illustrates many of these points.

A boy, aged 13 years, was kicked in the face by a horse about six years ago. The result of this accident was the formation of a lacrymal abscess and fistula. About a year after the accident he came under my care suffering from chronic dacryocystitis and lacrymal fistula. The treatment adopted consisted of first syringing out the sac ; afterwards the lower canaliculus was slit and the nasal duct was probed. The edges of the fistula were curetted and on the establishment of drainage into the nose the fistula healed. The sac, however, continued to secrete pus, but the patient, refusing further treatment, ceased to attend and did not come under my care again till December, 1907. When I saw him then the lacrymal sac was secreting pus freely, the upper lid was covered with velvety granulations, the conjunctiva was intensely injected, and the cornea was inflamed, vessels beginning to extend into its substance, forming pannus. Great photophobia and lacrymation were prominent symptoms. Conjunctivitis and keratitis were also present in the other eye. The conjunctiva was washed many times daily with boric acid lotion, and the lacrymal sac was syringed out with chinosol solution 1 in 4000 . This treatment was only kept up for a few days, as the boy again became impatient, and on Jan. 2nd the operation of excision of the lacrymal sac was performed. The mucous membrane along the upper edge of the tarsal cartilage of the upper lid was first excised with scissors in order to diminish the vascular supply to the granulations on the eyelid. The usual antiseptic precautions were observed and chloroform having been administered an incision about one and a half inches in length, commencing well above the tendo oculi, was carried downwards, and then round the inner third of the inferior margin of the orbit. The sac, which was easily recognised by its bluish-white colour, was cleared; the tendo oculi being divided, the sac was then separated from the bone beneath by means of the knife and curved scissors. The whole sac was then carefully dissected out with knife and scissors, adrenalin solution being employed to arrest hæmorrhage. Owing to cicatricial adhesions a small portion of the sac was torn off and left behind; this was afterwards dissected away and the wound was freely curetted. The canaliculi were cut through and the nasal duct, as far as possible, separated with fine scissors and knife from the lacrymal canal, and the upper part of the nasal duct divided. The wound was stitched up with fine silk, including the skin and tendo oculi with its fibrous expansion, and a small gauze drain left in the lower angle. As the eyelids were swollen and puffy for a day or two after the operation the gauze drain was kept in for three or four days. There was a certain amount of suppuration in the track of the drain but the temperature never rose above $99^{\circ} \mathrm{F}$. and in about a fortnight the wound was soundly healed. The inflammation of the conjunctivæ and cornex gradually subsided, and when last seen, excepting for the slight scar, the eye had quite its normal appearance. There was no weakening of the lids in consequence of the division of the tendo oculi, the latter having been carefully stitched. There was no epiphora, except when caused by emotion or any unusual irritation.

Bibliography.-Norris and Oliver: System of Diseases of the Fye Juler: Ophthalmic Science and Practice. George A. Berry: Disease of the Eye. Grimsdale and Brewerton: Ophthalmic Operations. Haab: Operative Ophthalmology.

Pietermaritzburg, Natal.

\section{Clinital atotes:}

\section{MEDICAL, SURGICAL, OBSTETRICAL, AND THERAPEUTICAL.}

\section{A NOTE ON LATENT DYSENTERY IN CENTRAL AFRIOA.}

By Hugh S. Stannus, M.B. Lond., MEDICAL OFFYCER, NYASALAYD.

Is a paper read before the Royal Medical and Chirurgical Society, and published in the Practitioner for June, 1907, entitled "The Early Diagnosis and Cure of the Presuppurative Stage of Amœbic Hepatitis," Major Leonard Rogers, I.M.S., described a series of cases in India presenting symptoms which he has shown are associated with the hepatitis of amobic dysentery before abscess formation and which are capable of cure by ipecacuanha. It is with the idea of drawing the attention of those practising in Africa to the fact that this class of case may be met with in this continent that $I$ give a short account of a case occurring in Nyasaland.

A man, single, aged 30 years, was admitted to hospital on Nov. 13th, 1907, with a temperature of $101 \cdot 8^{\circ} \mathrm{F}$. and a pulse-rate of 80 . He was depressed, weak and unable to walk. He gave a history of two weeks' fever, headache, malaise, with some looseness of the bowels. He had taken 20 grains of quinine per diem during the first 